\title{
Instrumentação interespinhosa na doença degenerativa da coluna lombar: medição da altura do disco no segmento instrumentado
} Interspinous instrumentation in patients with degenerative lumbar spine
disease: disc height measurement on instrumented segment
Instrumentación interespinosa en la enfermedad degenerativa de
la columna lumbar: medición de la altura del disco en el segmento
instrumentado

\author{
José Alberto de Castro Guimarães Consciência'
}

\section{RESUMO}

Introdução: na última década, a instrumentação interespinhosa vem sendo mais frequentemente utilizada. Apesar dos inúmeros artigos publicados em revistas internacionais de reconhecido mérito científico, são escassas as referências à modificação da altura do disco no segmento tratado, secundária àquela instrumentação. Objectivo: quantificar uma eventual modificação da altura discal decorrente da aplicação de instrumentação interespinhosa (DIAM - CousinBiotech - Medtronic Sofamor Danek $\mathrm{Inc}^{\complement}$ ). Métodos: o autor avalia um grupo de 20 pacientes com patologia degenerativa da coluna lombar e os seguintes critérios de inclusão: idade $>40$ e $<80$; índice de massa corporal $<40$; dor visual analogue scale (VAS) $\geq 6$; Oswestry Disability Index (ODI) $>30$; Zung Depression Rating Scale $<39$; Modified Somatic Perception Questionnaire (MSPQ) $<15$; degenerescência vertebral tipo 2 e 3 da classificação de Benzel e discal tipo 3 e 4 da classificação de Pfirrmann. Utilizando uma fórmula matemática, e um

\section{ABSTRACT}

Introduction: the use of interspinous instrumentation has been increasing in the last decade. However, in spite of the numerous papers seen in relevant scientific publications, there are very few references to an eventual disc height variation in the instrumented segment. Objective: to certify eventual changes in disk height after interspinous instrumentation (DIAM-Cousin-Biotech-Medtronic Sofamor Danek $\left.I n c^{\mathcal{O}}\right)$. Methods: the author evaluated 20 patients with degenerative lumbar disease and the following criteria: age $>40$ and $<80$; body mass index <40; pain visual analogue scale $\geq 6$; Oswestry Inability Index $>30$; Zung Depression Rating Scale <39; Modified Somatic Perception Questionnaire $<15$; degenerative bone disease grade 2 and 3 according to Benzel classification and degenerative disk disease grade 3 and 4 according to Pfirmann classification. Using a special formula and a computer image program (Adobe Photoshop 9.0 CS2), side radiograms of the lumbar

\section{RESUMEN}

Introducción: la instrumentación interespinosa tiene sido empleada con creciente frecuencia en la última década. Pero, apesar de las numerosas publicaciones cientificas hechas en jornales cientificos de reconocido mérito internacional, se han producido muy escasas referencias a una hipotética modificación de la altura discal dependiente de la técnica. Objetivo: cuantificar una eventual modificación de la altura discal inherente a la aplicación de instrumentación interespinosa. Métodos: el autor hace una evaluación de un grupo de 20 pacientes con patología degenerativa de la columna lumbar, y diversos criterios de inclusión, a saber: edad $>40$ y <80; índice de masa corpórea $<40$; dolor Visual Analogue Scale $\geq 6$; Oswestry Disability Index >30; Zung Depression Rating Scale $<39$; Modified Somatic Perception Questionnaire $<15$; patología degenerativa ósea lumbar de tipo 2 y 3 de la clasificación de Benzel y discal de tipo 3 y 4 de la clasificación de Pfirmann. Empleando una fórmula mate-

\footnotetext{
Trabalho realizado na Faculdade de Ciências Médicas de Lisboa - Lisboa, Portugal.

'MD, PhD, Professor de Ortopedia da Faculdade de Ciências Médicas de Lisboa; Director do Serviço de Ortopedia do Hospital de S. Francisco Xavier - CHLO Lisboa, Portugal.

Extracto parcial da tese de Doutoramento do autor intitulada "Estabilização dinâmica em Patologia Degenerativa da Coluna Lombar - Estado da Arte e Contributo Pessoal". 
programa computorizado de imagem (Adobe Photoshop 9.0 CS2), foram efectuadas medições em radiografias da coluna lombar obtidas na incidência de perfil em posição ortostática. A amplificação utilizada para esses exames foi de $70 \%$, tendo sido comparados os valores obtidos no pré-operatório com os referentes a dois anos após a cirurgia. Resultados: foi constatado um aumento global em média de $1,53 \mathrm{~mm}$ com desvio padrão de 1,09 mm e $\mathrm{p}=0,0002$, quando avaliadas as diferenças decorrentes da aplicação da referida fórmula. Contudo, quando foi analisada a variação anterior e posterior separadamente, observou-se diferença média superior $(0,45 \mathrm{~mm})$ na variação da distância intervertebral posterior $(\mathrm{p}=0,0002)$ quando comparada com a anterior $(\mathrm{p}=0,001)$, o que indicia um ligeiro efeito cifosante da instrumentação. Conclusões: nos casos de doença degenerativa lombar de tipo 2/3 de Benzel e 3/4 de Pfirmann, a aplicação de sistemas interespinhosos poderá proporcionar uma modificação da altura do disco intervertebral, aumentando-o ligeiramente, o que inevitavelmente influenciará as dimensões do canal vertebral.

spine in orthostatic position and with $70 \%$ amplification were obtained presurgery and two years after surgery. Results: after applying the mentioned formula, the results showed an increased global disk height average $1.53 \mathrm{~mm}$, with a standard deviation of $1.09 \mathrm{~mm}$ and $p=0.0002$. However, there was a large increase $(0.45 \mathrm{~mm})$ in posterior disk height $(p=0.0002)$ when compared with anterior measurement ( $p=0.001$ ) pointing out a slight kyphosing effect related to the instrumentation. Conclusions: in Benzel grade 2 and 3 and Pfirmann grade 3 and 4 degenerative lumbar disease, the use of an interspinous system seemed to be able to increase disk height and to improve neural canal dimensions.

KEYWORDS: Spine; Intervertebral disk; Biomechanics; Orthopedic fixation devices; Lumbar vertebrae/surgery mática y recorriendo a un programa computarizado de imagen (Adobe Photoshop 9.0 CS2) se efectuaran las mediciones en radiografias de la columna lumbar, en incidencia de perfil y posición ortogonal. La amplificación empleada ha sido de 70\% se comparando los valores obtenidos antes con los referentes a dos años después de la cirugía. Resultados: se verificó un aumento global en media de 1,53 mm con un desvío padrón de 1,09 mm y $p=0,0002$, cuando se evaluaran las diferencias inherentes a la aplicación de la mencionada fórmula. Sin embargo, cuando la variación anterior y posterior en separado fue verificada, había una diferencia media superior $(0,45 \mathrm{~mm})$ en la variación de la distancia intervertebral posterior $(p=0,0002)$ cuando comparada con la anterior $(p=0,001)$, lo que indicia un ligero efecto cifosante de la instrumentación. Conclusiones: en los casos de patología degenerativa lumbar de tipo 2 y 3 de Benzel $y$ discal de tipo 3 y 4 de Pfirmann, la aplicación de sistemas interespinosos parece proporcionar una modificación de la altura del disco intervertebral, lo aumentando y en consecuencia modificando las dimensiones del canal neural.

\section{INTRODUÇÃO}

Quando a patologia degenerativa lombar atinge os graus mais elevados de sua classificação, a solução terapêutica indicada é habitualmente a descompressão directa, muitas vezes associada a uma artrodese particularmente quando se confirma instabilidade significativa. Esta atitude tem sido validada por bibliografia convincente ${ }^{1-8}$ publicada sobre o assunto, e de facto também confirmada pela nossa prática clínica. No entanto, quando a doença degenerativa é apenas moderada, não parece tão linear a selecção da terapêutica adequada. Nestes casos, a descompressão directa das estruturas neurológicas pode ainda ser necessária, sugeriu-se que ela se obterá de forma indirecta ${ }^{9,10}$, como consequência de técnicas cirúrgicas seleccionadas.

A artrodese produz sempre uma restrição da mobilidade e uma consequente alteração da biomecânica segmentar que, em muitos casos, é considerada inadequada à patologia existente ${ }^{11-14}$. Nas fases precoces da doença degenerativa da coluna lombar, o recurso à estabilização dinâmica interespinhosa tem vindo a ser referido como uma das possibilidades terapêuticas ${ }^{15-19}$. Os primeiros trabalhos foram publicados nos anos $1950^{20}$, tendo Senegas et al. ${ }^{21}$, na década de 1980 , contribuído significativamente para o incremento da sua aplicação, bem como para o 
aumento do número de publicações que de algum modo procuraram estudar.

Em respeita à eventual variação da altura discal decorrente da sua aplicação, originadora de eventual descompressão indirecta foraminal e central, existem apenas referências a uma modificação da morfologia sagital do disco inerente à cifose local induzida ${ }^{22}$ ou à modificação da amplitude do canal central e foraminal ${ }^{23}$, mas não concretamente a uma possível modificação da altura discal.

\section{MÉTODOS}

Este estudo foi realizado numa série de 20 pacientes com o diagnóstico clínico de doença degenerativa lombar, seleccionados de acordo com diversos critérios de inclusão: idade entre 40 e 80 anos; índice de massa corporal (IMC) $<40$; dor lombar e/ou dos membros inferiores Visual Analogue Scale (VAS) $\geq 6$; Oswestry Disability Index (ODI) $>30$; doença degenerativa vertebral graus 2 e 3 da classificação de Benzel $^{24}$ e discal graus 3 e 4 da classificação de Pfirrmann ${ }^{25}$; ausência de instabilidade $>2 \mathrm{~mm}$; Zung Depression Rating Scale $<50^{26}$; Modified Somatic Perception Questionnaire (MSPQ) $<15^{27}$ terem efectuado Fisioterapia em local seleccionado durante seis ou mais meses e ausência de qualquer litígio laboral ou judicial.

No pré-operatório, foram efetuadas radiografias da coluna lombossagrada em ortoestatismo e nas incidências de ântero-posterior, perfil, hiperextensão e hiperflexão (exame dinâmico funcional), repetidas com a mesma técnica descrita, um e seis meses, um e dois anos após a data da cirurgia.

Estes exames permitiram a diferenciação gradativa da doença degenerativa vertebral lombar, de acordo com os critérios de Benzel ${ }^{24}$, e determinar quer a lordose lombar, quer possíveis sinais de instabilidade ao longo de todo o estudo. Ao existir, esta última deveria ser confirmada pela assimetria de posicionamento do alinhamento posterior dos muros vertebrais, no nível a tratar ou noutro, evidenciada nas imagens de alternância das posições de inclinação anterior e posterior da coluna lombar, desde que documentassem um deslizamento intervertebral superior a $2 \mathrm{~mm}$. Foi possível, também, confirmar se os pacientes teriam espondilolistese degenerativa até grau 1 de Meyerding, a única variante desta patologia admissível no presente estudo.

Ainda no pré-operatório, todos os pacientes realizaram uma ressonância magnética nuclear de coluna lombossagrada, a qual permitiu a confirmação das alterações degenerativas no segmento vertebral a tratar, e ainda a classificação gradativa da doença discal, de acordo com os critérios descritos por Pfirrmann et al. ${ }^{25}$.

Para determinação da variação da altura do disco no nível instrumentado, todos as imagens obtidas foram submitdas a um processo de digitalização e harmonização em programa informático comum (Adobe Photoshop CS2 9.0), que permitiu não só obter uma amplificação de $70 \%$ em todos os exames como ainda realizar as medições necessárias. Considerou-se, para efeitos da avaliação pretendida, que uma vez obtida essa igualização, as dimensões do corpo vertebral adjacente ao disco do segmento instrumentado teriam de ser sempre idênticas em duas coordenadas, comprimento e altura do corpo vertebral (Figura 1).

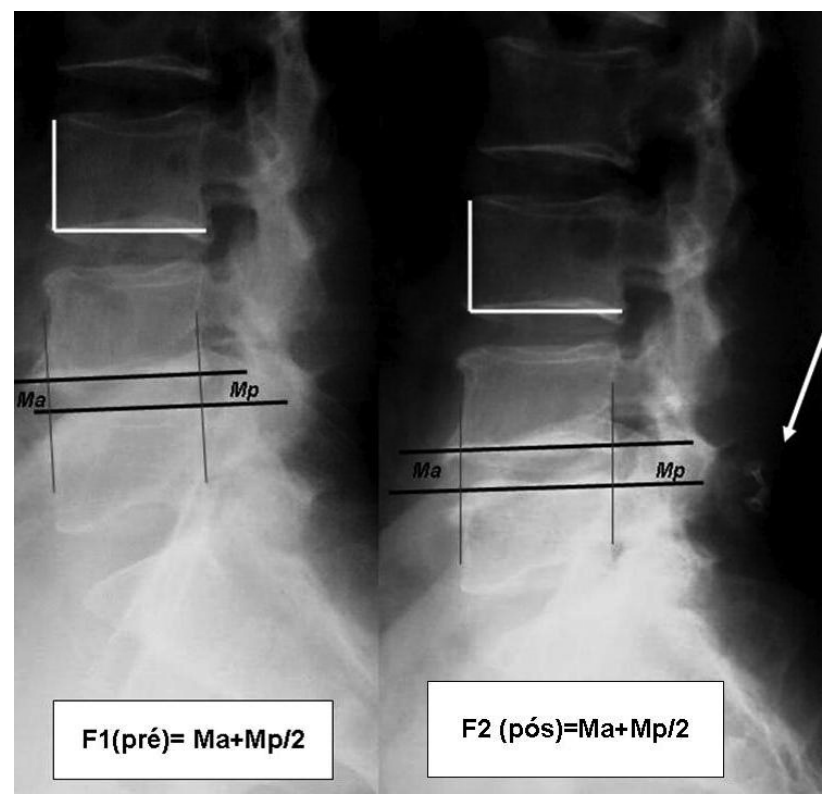

Adaptado de: Consciência JACG. "Estabilização dinâmica em patologia degenerativa da coluna lombar - "Estado da Arte" e contributo pessoal. Lisboa: Grafilinha; 2007.

Figura 7

Medição computorizada da diferença da altura do disco L4-L5 após aplicação de "DIAM" (seta)

A medição da altura do disco L4-L5 pré operatória e dois anos após a cirurgia foi calculada na incidência de perfil em carga (ortostatismo), após o referido procedimento, e obtida pela aplicação da fórmula:

$\mathrm{F}=\mathrm{Ma}+\mathrm{Mp} / 2$.

onde:

Ma: representa a distância entre os vértices do ângulo ântero-inferior e ântero-superior do corpo das vértebras adjacentes ao disco a medir;

Mp: a distância entre os ângulos póstero-inferior e pósterosuperior, respectivamente, dos mesmos corpos vertebrais.

Todos os pacientes foram operados em 2005 pelo mesmo cirurgião que efectuou a aplicação do dispositivo interespinhoso DIAM (Cousin-Biotech - Medtronic Sofamor Danek Inc ${ }^{\circ}$ - USA). A técnica foi realizada após desinserção sublaminar do ligamento amarelo e remoção integral do ligamento interespinhoso, mas com preservação cuidadosa do ligamento supraespinhoso, bem como o seu tensionamento apropriado. Em 55\% dos pacientes, a instrumentação foi aplicada por meio de abordagem tubular unilateral previamente utilizada para descompressão foraminal. 
O tratamento estatístico dos dados foi realizado com apoio especializado, tendo sido determinada a média e o desvio padrão dos valores globais correspondentes às medições realizadas no pré-operatório e após a cirurgia, bem como a diferença resultante entre elas. Uma vez que esses dados não aparentavam uma distribuição Gaussiana, utilizou-se o teste não paramétrico dos postos sinalizados de Wilcoxon para amostras emparelhadas.

\section{RESULTADOS}

A Tabela 1 apresenta os valores em "mm" das diferentes medições realizadas nos discos L4-L5 de todos os pacientes, de forma a poder comparar o pré-operatório com dois anos após a cirurgia. Refira-se, como já mencionado, que só foram avaliadas radiografias da coluna lombar na incidência de perfil e na posição ortogonal.

A1 e A2 representam, respectivamente, a altura discal anterior pré e dois anos após cirurgia, P1 e P2 a altura discal posterior pré e dois anos após cirurgia, sendo F1 e F2 os valores decorrentes da aplicação da fórmula de medição anteriormente descrita pré e dois anos após cirurgia.
Em três dos discos não foram encontradas quaisquer diferenças entre as duas medições, no entanto aumentaram: 0,5 mm, um paciente; $1 \mathrm{~mm}$, sete pacientes; $1,5 \mathrm{~mm}$, um paciente; $2 \mathrm{~mm}$, dois pacientes; $2,5 \mathrm{~mm}$, dois pacientes; $3 \mathrm{~mm}$, três; $3,5 \mathrm{~mm}$, um paciente.

Na Tabela 2 apresentam-se também os valores da média e desvio padrão, correspondentes aos conjuntos das diferentes medições efectuadas.

Os valores calculados demonstraram existir globalmente um aumento em média de $1,53 \mathrm{~mm}$ com desvio padrão de $\pm 1,09 \mathrm{~mm}$. Contudo, torna-se evidente que a média da diferença da altura discal posterior é ligeiramente superior à anterior $(0,45 \mathrm{~mm})$.

Ressalta-se que nenhum disco diminuiu globalmente a sua altura, sendo pequena a diferença entre os valores obtidos. No entanto, recorde-se que estamos a avaliar uma estrutura cuja altura máxima ronda os $9 \mathrm{~mm}$, e que aquele valor corresponde a uma ampliação de apenas 70\%.

$\mathrm{Na}$ análise estatística, assumido o nível de significância de 0,05 (95\%), foi efectuado o teste não paramétrico dos postos sinalizados de Wilcoxon para amostras

TABELA 1 - Resultados da determinação das diferenças (pré e dois anos pós-operatórios) da altura discal (L4-L5) de todos os pacientes

\begin{tabular}{lccccccccc}
\hline Paciente & A1 & P1 & F1 & A2 & P2 & F2 & A2-A1 & P2-P1 & F2-F1 \\
\hline A & 4 & 3 & 3,5 & 5 & 5 & 4,5 & 1 & 2 & 1 \\
B & 6 & 6 & 6 & 6 & 6 & 6 & 0 & 0 & 0 \\
C & 5 & 2 & 3,5 & 7 & 5 & 6 & 2 & 3 & 2,5 \\
D & 4 & 2 & 4 & 4 & 2 & 4 & 0 & 0 & 0 \\
E & 4 & 2 & 3 & 7 & 6 & 6,5 & 3 & 4 & 3,5 \\
F & 5 & 3 & 4 & 4 & 6 & 5 & -1 & 3 & 1 \\
G & 7 & 4 & 5,5 & 8 & 6 & 7 & 1 & 2 & 1,5 \\
H & 6 & 3 & 4,5 & 9 & 6 & 7,5 & 3 & 3 & 3 \\
I & 5 & 3 & 4 & 8 & 4 & 6,5 & 3 & 1 & 2,5 \\
J & 7 & 3 & 5 & 7 & 5 & 6 & 0 & 2 & 1 \\
K & 11 & 4 & 7,5 & 12 & 5 & 8,5 & 1 & 1 & 1 \\
L & 6 & 2 & 4 & 6 & 2 & 4 & 0 & 0 & 0 \\
M & 5 & 3 & 4 & 6 & 4 & 5 & 1 & 1 & 1 \\
N & 5 & 3 & 4 & 6 & 4 & 5 & 1 & 1 & 1 \\
O & 4 & 4 & 4 & 6 & 6 & 6 & 2 & 2 & 2 \\
P & 7 & 5 & 6 & 8 & 8 & 8 & 1 & 3 & 2 \\
Q & 11 & 6 & 8,5 & 11 & 7 & 9 & 0 & 1 & 0,5 \\
R & 4 & 2 & 3 & 7 & 5 & 6 & 3 & 3 & 3 \\
S & 6 & 3 & 4,5 & 10 & 5 & 7,5 & 4 & 2 & 3 \\
T & 4 & 4 & 4 & 5 & 5 & 5 & 1 & 1 & 1 \\
\hline
\end{tabular}

Valores expressos em milímetros. A2 e A1: diferença da altura discal anterior dois anos após e pré-cirurgia; P2 e P1: diferença da altura discal posterior dois anos após e pré-cirurgia; F2 e F1: valores decorrentes da aplicação da fórmula de medição ( $F=M a+M p / 2)$ dois anos após e pré-cirurgia.

TABELA 2 - Valores das diferentes medições da altura do disco intervertebral no pré e dois anos pós-operatório (raio $\mathrm{x}$ de perfil)

\begin{tabular}{lccccccccc}
\hline & A1 & P1 & F1 & A2 & P2 & F2 & A2-A1 & P2-P1 & F2-F1 \\
\hline Média & 5,8 & 3,35 & 4,63 & 7,1 & 5,1 & 6,15 & 1,3 & 1,75 & 1,53 \\
Desvio padrão & 2,07 & 1,23 & 1,43 & 2,15 & 1,45 & 1,43 & 1,35 & 1,16 & 1,09 \\
\hline
\end{tabular}

Valores expressos em médias e desvios padrão. A2 e A1: diferença da altura discal anterior dois anos após e pré-cirurgia; P2 e P1: diferença da altura discal posterior dois anos após e pré-cirurgia; $F 2$ e F1: valores decorrentes da aplicação da fórmula de medição $(F=M a+M p / 2)$ dois anos após e pré-cirurgia. 
emparelhadas tendo-se obtido para a variação A1-A2 (altura discal anterior pré e dois anos após cirurgia) $\mathrm{p}=0,001$, para P1-P2 (altura discal posterior pré e dois anos após cirurgia) um $\mathrm{p}=0,0002$ e para F1-F2 (valores decorrentes da aplicação da fórmula de medição pré e dois anos após cirurgia) um $\mathrm{p}=0,0002$.

$\mathrm{Na}$ Tabela 3 é possível ver os resultados da análise estatística realizada e já descrita, verificando-se uma diferença estatisticamente significativa entre os diferentes valores obtidos, ou seja, do pré-operatório para dois anos após a cirurgia.

\section{TABELA 3 - Resultados da aplicação do teste dos postos sinalizados de Wilcoxon para a análise comparativa das amostras emparelhadas

\begin{tabular}{lc}
\hline Variação das diferentes medições & Valor de $\mathrm{p}$ \\
\hline Diferença anterior (A2-A1) & 0,001 \\
Diferença posterior (P2-P1) & 0,0002 \\
Diferença após aplicação da fórmula (F2-F1) & 0,0002 \\
\hline
\end{tabular} \\ Significância $p \leq 0,05$.}

No Gráfico 1, são ainda apresentados os "boxplots" inerentes aos valores da análise estatística, sendo evidente que a diferença entre os valores tende a ser positiva o que indica que a altura discal após a cirurgia é superior a do pré-operatório.

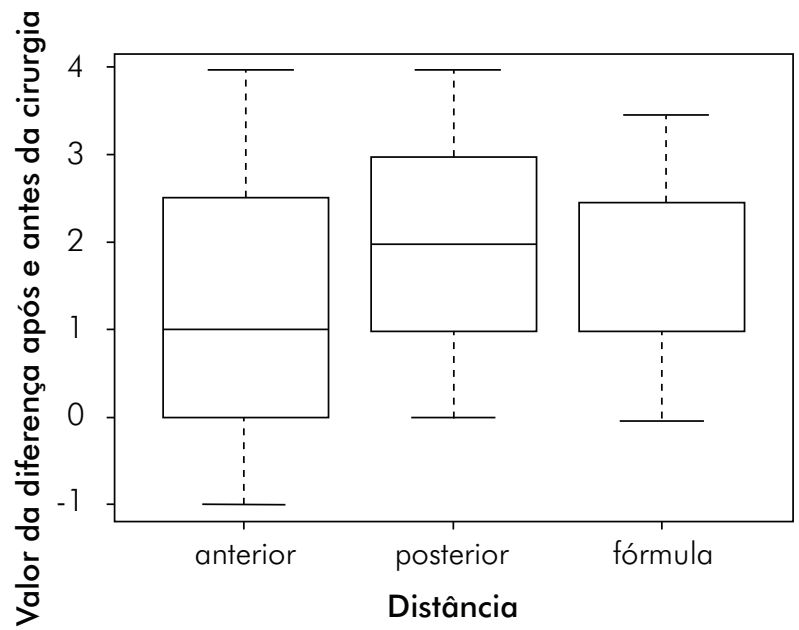

Gráfico 1

Expressão gráfica (boxplots) da análise estatística efectuada com o teste de Wilcoxon para amostras emparelhadas.

\section{DISCUSSÃO}

Os efeitos biomecânicos decorrentes da aplicação de espaçadores interespinhosos em patologia degenerativa lombar, e a sua correspondente avaliação, têm merecido o interesse de diferentes autores, mas pouco se tem dito sobre uma eventual variação da altura do disco secundária à sua aplicação.

Assim, Lindsey et al. ${ }^{28}$ constatam uma ligeira diminuição da amplitude da flexão extensão após aplicação desses dispositivos, e Swanson et al. ${ }^{29}$ referem que a pressão intradiscal decresce no nível instrumentado, mas não se altera nos segmentos adjacentes. Wiseman et al. ${ }^{30}$, por outro lado, confirmam que estes sistemas actuam biomecanicamente modificando as forças exercidas no arco neural, e descreve pormenorizadamente a sua acção descompressora facetária, chegando mesmo a quantificar esse decréscimo de pressão.

Kim et al..$^{22}$ estudaram, especificamente, a variação da altura do disco numa série de pacientes com patologia degenerativa lombar, operados com este tipo de instrumental, mas não encontrou qualquer alteração. $\mathrm{O}$ grau de degenerescência lombar preexistente é omitido, prejudicando deste modo a interpretação final dos seus resultados.

No presente grupo de pacientes, as medições realizadas nas radiografias de perfil em posição ortostática, referentes às diferenças pré e pós-operatórias da altura do disco no segmento intervencionado (L4-L5), revelaram em média um aumento de $1,53 \mathrm{~mm}$, com um desvio padrão de \pm $1,09 \mathrm{~mm}$, e $\mathrm{p}=0,0002$. Porém, a análise pormenorizada do valor da média das diferenças obtidas para a altura discal anterior $(\mathrm{p}=0,001)$ e posterior $(\mathrm{p}=0,0002)$ no pré e pósoperatório revelou ser ligeiramente superior $(0,45 \mathrm{~mm})$ na medição posterior, o que é revelador de um efeito cifosante já descrito por Kim et al. ${ }^{22}$.

Foi verificado, ainda, que em nenhum disco se registrou uma diminuição de altura, permanecendo inalterados apenas três.

As diferenças globais foram calculadas pela metade do somatório da altura anterior e posterior do espaço discal $(\mathrm{F}=\mathrm{Ma}+\mathrm{Mp} / 2)$, sendo que o valor final obtido representa, portanto, um aumento médio da altura do disco de cerca de $35 \%$, o que nos parece considerável, sobretudo ponderando a amplificação utilizada (70\%).

Este facto, implicando um redimensionamento segmentar e consequente descompressão indirecta das estruturas neurológicas, constitui um efeito benéfico cuja tradução clínica já foi comentada, e nos parece importante realçar. Lee et al. ${ }^{23}$ e Richards et al. ${ }^{31}$ relataram apenas um acréscimo das dimensões foraminais e canalares centrais relacionadas com este tipo de instrumentação, e embora se possa suspeitar de certa influência sobre a altura discal, nada referiram concretamente quanto à sua existência.

É ainda interessante referir que os aumentos mais significativos da altura do espaço discal foram medidos em pacientes com patologia degenerativa lombar grau 3, segundo Benzel ${ }^{24}$, e grau 4 segundo Pfirrmann et al. ${ }^{25}$, ou seja, situações de gravidade moderada e ainda com alguma mobilidade segmentar, sugerindo constituírem formas clínicas favoráveis a esta indicação terapêutica. Aliás, é importante salientar que esta constatação está de acordo com o que anteriormente havia sido referido por Sénégas ${ }^{19}$ e Caserta et al. ${ }^{15,32}$, podendo também explicar os resultados menos favoráveis de Kim et al. ${ }^{22}$.

Por último, não foram encontradas outras referências ao aumento da altura do espaço discal secundário à 
instrumentação interespinhosa lombar, em publicações de revistas internacionais de referência.

\section{CONCLUSÃO}

Quando aplicados no tratamento de patologia degenerativa da coluna lombar do tipo referido neste estudo, os sistemas interespinhosos semirígidos influenciaram positivamente a altura do disco instrumentado, revelando assim um potencial de redimensionamento foraminal e central inerente à modificação produzida. Este efeito revela-se mais acentuado quando a degenerescência lom- bar é de tipo 3 de Benzel e 4 de Pfirmann, o que de algum modo as constitui indicações preferenciais para esta opção cirúrgica.

Acresce ainda que a acção biomecânica produzida origina uma ligeira cifose localizada no nível instrumentado.

\section{AGRADECIMENTOS}

Agradecimentos ao doutor Nuno Spulveda, professor de estatística da Escola Superior Egas Moniz em Almada, Portugal, o qual é responsável pela análise estatística dos dados apresentados.

\section{REFERÊNCIAS}

1. Hansraj KK, Cammisa FP Jr, O’Leary PF, Crockett HC, Fras CI, Cohen MS, et al. Decompressive surgery for typical lumbar spinal stenosis. Clin Orthop Relat Res. 2001;(384):10-7.

2. Kostuik JP. Controversies in cauda equina syndrome and lumbar disc herniation. In: Wiesel SW, Weinstein JN, Herkowitz H, et al., editors. The lumbar spine. 2nd ed. Philadelphia: Saunders;1996. p. 582-7.

3. Malmivaara A, Slätis P, Heliövaara M, Sainio P, Kinnunen H, Kankare J, Dalin-Hirvonen N, Seitsalo S, Herno A, Kortekangas P, Niinimäki T, Rönty H, Tallroth K, Turunen V, Knekt P, Härkänen T, Hurri H; Finnish Lumbar Spinal Research Group. Surgical or nonoperative treatment for lumbar spinal stenosis? A randomized controlled trial. Spine (Phila Pa 1976). 2007;32(1):1-8.

4. Postacchini F, Cinotti G, Perugia D, Gumina S. The surgical treatment of central lumbar stenosis. Multiple laminotomy compared with total laminectomy. J Bone Joint Surg Br. 1993;75(3):386-92.

5. Deyo RA, Nachemson A, Mirza SK. Spinal-fusion surgery - the case for restraint. N Engl J Med. 2004;350(7):722-6.

6. Carragee E. The role of surgery in low back pain. Curr Orthop. 2007;21(1):916.

7. Azevedo C, Consciência JG. Biomecânica da fusão intervertebral lombar. Rev Port Ortop Traumatol. 2005;13(3):25-36.

8. Brantigan JW, Steffee AD. Lumbar fusion and stabilization. In: Yonenobu K, Ono K, Takemitsu Y. Lumbar fusion and stabilization. Tokyo: Springer; 1993. p.126.
9. Taylor J, Pupin P, Delajoux S, Palmer S. Device for intervertebral assisted motion: technique and initial results. Neurosurg Focus. 2007;22(1):E6.

10. Taylor J. Biomechanical requirements for the posterior control of the center of rotation - Fundamental principles \& techniques in non-fusion solutions [oral presentation]. Paris, 2005 Jun.

11.Cherkin DC, Deyo RA, Loeser JD, Bush T, Waddell G. An international comparison of back surgery rates. Spine (Phila Pa 1976). 1994;19(11):1201-6.

12. Birkmeyer JD, editor. The dartmouth atlas of musculoskeletal health care. Chicago: AHA Press; 2000.

13. Volinn E, Mayer J, Diehr P, Van Koevering D, Connell FA, Loeser JD. Small area analysis of surgery for low-back pain. Spine (Phila Pa 1976). 1992;17(5):575-81.

14. Irwin ZN, Hilibrand A, Gustavel M, McLain R, Shaffer W, Myers M, et al. Variation in surgical decision making for degenerative spinal disorders. Part I: lumbar spine. Spine (Phila Pa 1976). 2005;30(19):2208-13.

15.Caserta S, Misaggi B, Peroni D, La Maida GA. Elastic stabilization combined with rigid fusion: a prevention of pathology of the border area. Proceedings of the 24th National Congress of the Italian Spine Society. Eur Spine J. 2001;10(4):352-62.

16. Christie SD, Song JK, Fessler RG. Dynamic interspinous process technology. Spine (Phila Pa 1976). 2005;30(16 Suppl):S73-8.

17.Consciência JG. Fundamentals principles \& techniques in non fusion solutions [oral presentation]. Berlin, 2005 Sep 9-10.
18.Kaech DL, Jinkins JR. The interspinous «U”: a new restabilization device for the lumbar spine. In: Kaech DL, Jinkins JR, editors. Spinal restabilization procedures: diagnostic and therapeutic aspects of intervertebral fusion cages, artificial discs and mobile implants. Amsterdam; Boston; London: Elsevier Science; 2002. cap. 30, p. 355-62.

19.Sénégas J. Mechanical supplementation by non-rigid fixation in degenerative intervertebral lumbar segments: the Wallis system. Eur Spine J. 2002;11 Suppl 2:S164-9.

20.Knowles FL. The Knowles vertebral support operation. J Iowa State Med Soc. 1958;48(10):551-4.

21.Senegas J, Etchevers JP, Vital JM, Baulny D, Grenier F. [Recalibration of the lumbar canal, an alternative to laminectomy in the treatment of lumbar canal stenosis]. Rev Chir Orthop Reparatrice Appar Mot. 1988;74(1):15-22. French.

22.Kim KA, McDonald M, Pik JH, Khoueir P, Wang MY. Dynamic intraspinous spacer technology for posterior stabilization: case-control study on the safety, sagittal angulation, and pain outcome at 1-year followup evolution. Neurosurg Focus. 2007;22(1):E7.

23.Lee J, Hida K, Seki T, Iwasaki Y, Minoru A. An interspinous process distractor (X STOP) for lumbar spinal stenosis in elderly patients: preliminary experiences in 10 consecutive cases. J Spinal Disord Tech. 2004;17(1):72-7; discussion 78.

24.Benzel E. Spine surgery. Philadelphia: Churchill Livingstone; 1999.

25.Pfirrmann CW, Metzdorf A, Zanetti M, Hodler J, Boos N. Magnetic resonance classification of lumbar intervertebral disc degeneration. Spine (Phila $\mathrm{Pa}$ 1976). 2001;26(17):1873-8. 
26.Zung WW. A self-rating depression scale. Arch Gen Psyquiatry. $1965 ; 12: 63-70$.

27.Main CJ. The Modified Somatic Perception Questionnaire (MSPQ). J Psychosom Res. 1983;27(6):503-14.

28.Lindsey DP, Swanson KE, Fuchs P, Hsu KY, Zucherman JF, Yerby SA. The effects of an interspinous implant on the kinematics of the instrumented and adjacent levels in the lumbar spine. Spine (Phila Pa 1976). 2003;28(19):2192-7.

29. Swanson KE, Lindsey DP, Hsu KY, Zucherman JF, Yerby SA. The effects of an interspinous implant on intervertebral disc pressures. Spine (Phila Pa 1976). 2003;28(1):26-32.
30. Wiseman CM, Lindsey DP, Fredrick AD, Yerby SA. The effect of an interspinous process implant on facet loading during extension. Spine (Phila Pa 1976). 2005;30(8):903-7.

31.Richards JC, Majumdar S, Lindsey DP, Beaupré GS, Yerby SA.

The treatment mechanism of an interspinous process implant for lumbar neurogenic intermittent claudication. Spine (Phila Pa 1976). 2005;30(7):744-9.
32.Caserta S, La Maida G, Misaggi B, Peroni D, Pietrabissa R, Raimondi MT, et al. Elastic stabilization alone or combined with rigid fusion in spine surgery: a biomechanical study and clinical experience based on 82 cases. Eur Spine J. 2002;11 Suppl 2:S192-7.

\section{Correspondência:}

José Alberto de Castro Guimarães Consciência

Estrada do Forte do Alto de Duque CEP: 1495-005 - Lisboa, Portugal

Fone: + 351210431071

Secretária: +351210431065

E-mail: jconsciencia@chlo.min-saude.pt 